\title{
Fluid rim sign: a new ultrasonographic sign of soft tissue aspergillosis
}

\author{
Erhan Akpınar, Veysel Atilla Ayyıldız, İbrahim Petekkaya, Bülent Erbil, Vikram Dogra
}

\section{ABSTRACT}

The air crescent sign is a well-known important diagnostic finding in invasive pulmonary aspergillosis. Herein we report a distinctive but rare ultrasonographic appearance in a patient with myositis secondary to Aspergillus flavus infection, which can be considered as the soft tissue counterpart of the air crescent sign.
From the Departments of Radiology (E.A. $₫$ erhan.akpinar $@$ gmail.com, V.A.A.), Medical Oncology (I.P.), and Emergency Medicine (B.E.), Hacettepe University School of Medicine, Ankara, Turkey; the Department of Imaging Sciences (V.D.), University of Rochester Medical Center, Rochester, New York, USA.

Received 13 July 2012; revision requested 23 July 2012; revision received 28 August 2012; accepted 31 August 2012.

Published online 6 December 2012 DOI 10.4261/1305-3825.DIR.6384-12.1
$\mathbf{T}$ he air crescent sign is a well-known imaging characteristic of invasive pulmonary aspergillosis. It is recognized as a crescent-shaped or circumferential area of radiolucency within a parenchymal consolidation or nodular opacity. The nodular opacity represents the devitalized tissue, while the radiolucent area corresponds to the air filling the space between the necrotic tissue and surrounding parenchyma (1). Herein we report an unusual ultrasonographic appearance of musculoskeletal aspergillosis, which could be considered as the soft tissue counterpart of air crescent sign.

\section{Case report}

A 28-year-old patient with diagnosis of T-cell acute lymphoblastic leukemia presented with swelling and hyperemia on the thoracoabdominal wall, consistent with an infectious process. Laboratory studies revealed an increase in white blood cell count with a predominance of neutrophils (42 200/ $\mathrm{mm}^{3}$ ) and a C-reactive protein level of $6.86 \mathrm{mg} / \mathrm{dL}$. Axial contrast enhanced computed tomography (CT) scan of the upper abdomen showed a peripherally enhancing $26 \times 34 \mathrm{~mm}$ soft tissue lesion (Fig. 1a). Chest CT showed two parenchymal nodules in the left upper and lower lobes, one of which was surrounded by ground glass attenuation (Fig. 1b). Approximately ten days later, ultrasonography examination was performed with a broadband linear array probe (6-11 Mhz) (Toshiba iStyle, Xario, Toshiba Medical Systems, Tokyo, Japan) and revealed a $43 \times 34 \mathrm{~mm}$ heterogeneous lesion in the thoracoabdominal wall. The heterogeneous lesion contained a central $27 \times 21 \mathrm{~mm}$ hyperechoic solid component and a surrounding fluid component appearing as a rim that was $7 \mathrm{~mm}$ in thickness (Fig. 2a). Color Doppler examination did not detect any vascularity in the central component. The fluid component had internal echoes, with real-time examination showing the movement of these echoes. Compression with probe led to a change in the shape and thickness of the fluid rim (Fig. 2b), with no change in the central solid part. All of the imaging findings, together with the clinical presentation, were considered to be suggestive of invasive aspergillosis. A needle biopsy revealed purulent material. Pathological examination showed necrotic fat tissue along with septate fungal hyphae, which were verified as Aspergillus flavus in culture media. The patient was diagnosed as primary myositis secondary to A. flavus infection and responded well to antifungal therapy (liposomal amphotericin B) with disappearance of the soft tissue lesion.

\section{Discussion}

Invasive aspergillosis is an uncommon but life-threatening pathologic condition caused by Aspergillus organisms, and is mainly seen in immunocompromised patients $(2,3)$. Infection is secondary to inhalation of 

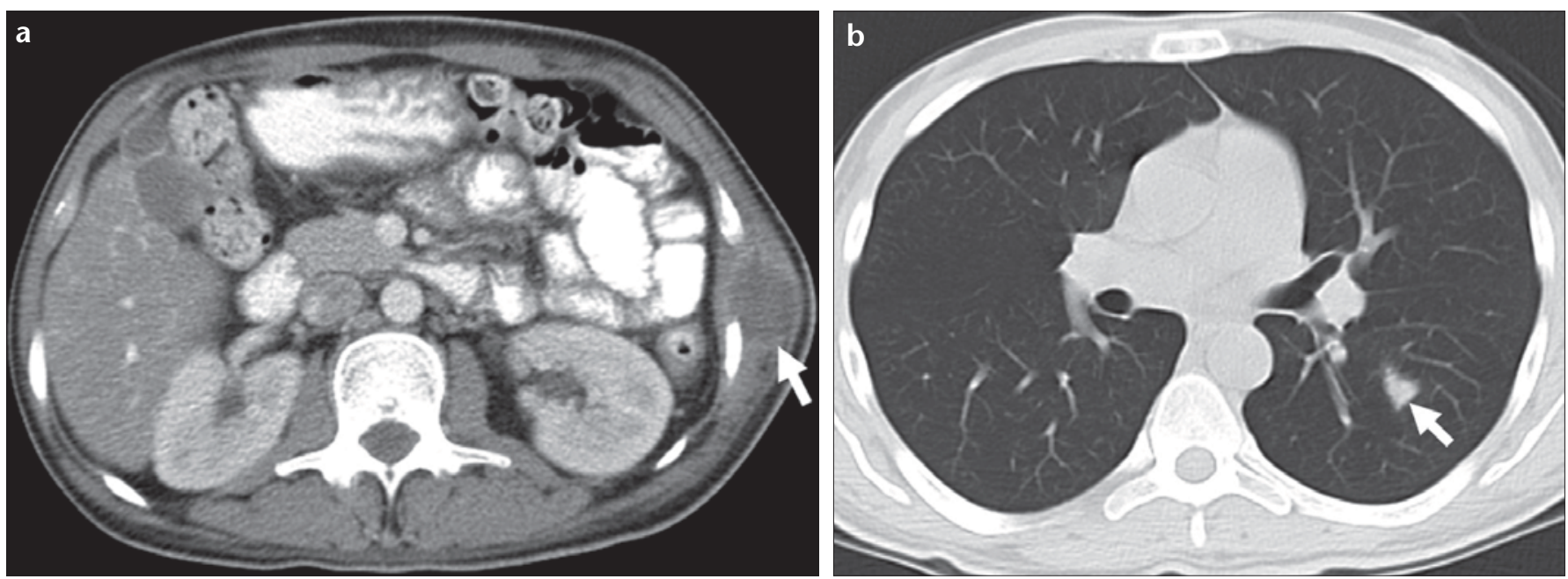

Figure 1. a, b. Axial contrast enhanced CT scan of the upper abdomen (a) shows peripherally enhancing soft tissue lesion (arrow) in the left side of thoracoabdominal wall. Chest CT scan (b) shows a parenchymal nodule surrounded by ground glass attenuation (arrow) in the superior segment of left lower lobe.
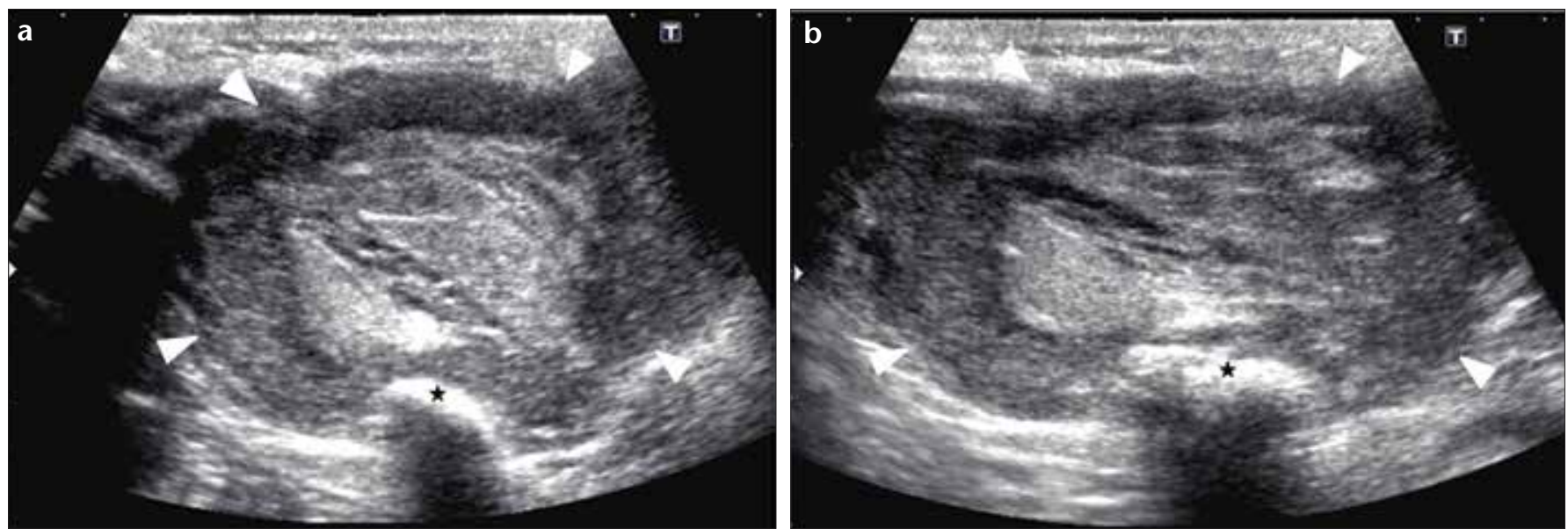

Figure 2. a, b. Ultrasonography image of the thoracoabdominal wall (a) shows central hyperechoic solid component surrounded by fluid rim (arrowheads). Ultrasonography image after compression (b) shows change in dimension and shape of the fluid rim (arrowheads). The rib appears as a bright hyperechoic line with intense acoustic shadowing (star) $(\mathbf{a}, \mathbf{b})$.

spores. Lung infection is characterized by angioinvasion with transbronchial spread of fungal hyphae into adjacent small to medium sized pulmonary arteries, resulting in arteriolar thrombosis and consequent hemorrhagic infarction of lung tissue. Contiguous spread of inflammatory masses and hematogenous dissemination of disease may occur (2-5). Disseminated infection occurs in $10 \%$ to $30 \%$ of patients with invasive pulmonary aspergillosis and may involve the gastrointestinal tract, skeletal system, brain and spinal cord, kidneys, liver, skin, sinuses, thyroid, spleen, heart, aorta, testes, and adrenals (3).

Infarction due to invasive aspergillosis and peripheral resorption of necrotic tissue by leukocytes results in an abscess cavity with necrotic solid component (1). If this cavity is formed within air-containing alveolar sacs or there is connection between airways, air can fill the abscess cavity. This results in the formation of the air crescent sign. However, when there is no communication with air-containing tissues or airways, as in the case of infarctions occurring within soft tissues, the cavity will be filled only with fluid and necrotic solid component. As the softtissue counterpart of the air-crescent sign is exceedingly rare and difficult to recognize, ultrasonographic examination is highly valuable in identifying the fluid rim and central solid echogenic part for such lesions in unique locations.

Ultrasonographic patterns of hepatic and splenic mycotic abscesses include the "wheel within wheel" phenomenon, "wagon wheel" appearance, "bull's eye" lesion, hypoechoic lesion, echogenic focus, and echogenic focus with an acoustic shadow. The "wheel within wheel" consists of a peripheral hypoechoic zone, an enclosed hyperechoic zone and a hypoechoic center, which is composed of pus, necrotic debris, and fungal elements. In the "wagon wheel" appearance, the middle echogenic layer is not continuous and resembles the shape of spokes. The identification of the "wheel within wheel" and/or "wagon wheel" appearance is more specific than the other imaging findings. Ultrasonographic imaging can also be used to follow-up antifungal therapy in hepatosplenic candidiasis (6-9).

In conclusion, ultrasonographic imaging may help in differential diagnosis, selection of patients for biopsy, and follow-up of response to antifungal therapy in the setting of 
musculoskeletal aspergillosis. The appearance of a necrotic core surrounded by air or fluid on radiologic studies, depending on the location of the lesion, might serve as an important diagnostic clue for aspergillosis.

\section{Conflict of interest disclosure}

The authors declared no conflicts of interest.

\section{References}

1. Abramson S. The air crescent sign. Radiology 2001; 218:230-232.

2. Franquet T, Müller NL, Giménez A, Guembe P, de La Torre J, Bagué S. Spectrum of pulmonary aspergillosis: histologic, clinical, and radiologic findings. Radiographics 2001; 21:825-837.
3. Thomas KE, Owens CM, Veys PA, Novelli $\mathrm{V}$, Costoli V. The radiological spectrum of invasive aspergillosis in children: a 10-year review. Pediatr Radiol 2003; 33:453-460.

4. Denning DW. Invasive aspergillosis. Clin Infect Dis 1998; 26:781-803.

5. Kuhlman JE, Fishman EK, Siegelman SS. Invasive pulmonary aspergillosis in acute leukemia: characteristic findings on CT, the CT halo sign, and the role of CT in early diagnosis. Radiology 1985; 157:611614.

6. Pastakia B, Shawker TH, Thaler M, O'Leary T, Pizzo PA. Hepatosplenic candidiasis: wheels within wheels. Radiology 1988; 166:417-421.

7. Grünebaum M, Ziv N, Kaplinsky C, Kornreich L, Horev G, Mor C. Liver candidiasis. The various sonographic patterns in the immunocompromised child. Pediatr Radiol 1991; 21:497-500.
8. Görg C, Weide R, Schwerk WB, Köppler $\mathrm{H}$, Havemann K. Ultrasound evaluation of hepatic and splenic microabscesses in the immunocompromised patient: sonographic patterns, differential diagnosis, and follow-up. J Clin Ultrasound 1994; 22:525-529.

9. Chew FS, Smith PL, Barboriak D. Candidal splenic abscesses. AJR Am J Roentgenol $1991 ; 156: 474$. 